\title{
Unexpected traqueal bronchus in a limbgirdle muscular dystrophy patient
}

Alexandre Pereira M., Carneiro S., Gonçalves C., Airosa I., Martins F., Arantes S. Hospital de Braga, Dept. of Anaesthesiology, Braga, Portugal

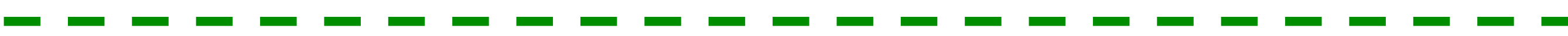

Background:

LimbGirdle Muscular Dystrophy (LGMD) is a myopathic disorder caused by alterations in genes required for normal muscle function ${ }^{1}$. Tracheal bronchus (Tb) is a congenital anomaly where the right upper lobe bronchus originates in the trachea rather than distal to the carina ${ }^{2}$. We describe the anesthetic management for thoracoscopic sympathectomy in a patient with LGMD type $2 \mathrm{~A}$ and an unknown Tb.
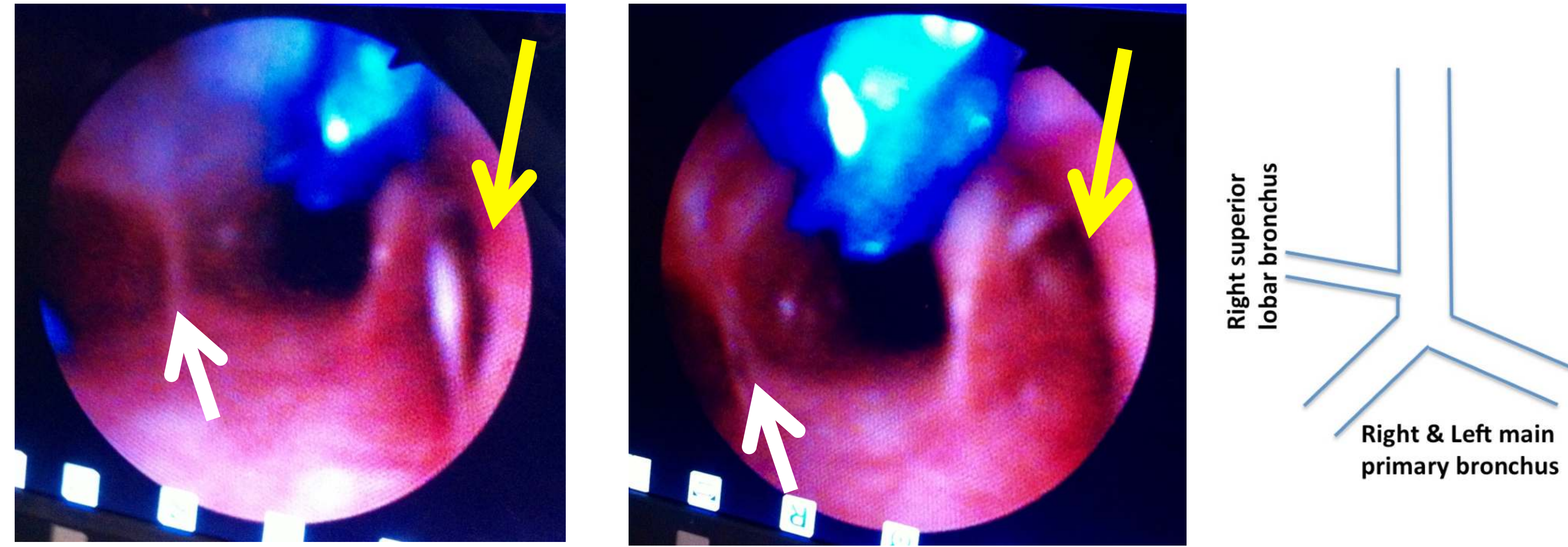

Figure 1,2: white arrow - Carina; Yellow arrow - traqueal bronchus

\section{Case Report:}

22 yr, male, ASA III, with LGMD manifested only by muscular features of the disease, scheduled for thoracoscopic sympathectomy. A Malignant Hyperthermia prevention ( $\mathrm{MH}$ ) protocol was used. The procedure was done under general intravenous anesthesia (propofol, remifentanil) and neuromuscular blockade (NMB) maintained with rocuronium. Monitorized with ASA standard plus anesthesia depth and NMB. After intubation with standard tracheal tube and surgical positioning, we inserted an EZBLOCKER ${ }^{\mathrm{TM}}$ under bronchoscopic guidance, during which we observed the presence of a Tb (Figure 1,2) that made lung isolation impossible using this device. Patient was repositioned and a 39Fr left double lumen tube placed. Surgical procedure was done uneventfully including the lung isolation techniques. Analgesia (intravenous and pleural local anesthetic instillation) and nausea and vomiting prophylaxis was done, and NMB reverted with sugammadex. Patient remained for $24 \mathrm{~h}$ in vigilance in the postanesthesia care unit and was discharged home inthe $5^{\text {th }}$ postoperative day.

Discussion:

LGMD has a prevalence ranging from $1: 45,000$ to $1: 123,000$. Two main types were described $(1,2)$, from which the type $2 \mathrm{~A}$ is the most common. Anesthetic management includes some critical points to consider: susceptibility to $\mathrm{MH}$; sensibility to sedative/hypnotics; possible airway management complications; high incidence of cardiac involvement; attention to type, dose, monitoring and adequate reversal of $\mathrm{NMB}^{1}$. $\mathrm{Tb}$ is a congenital anomaly with an incidence from $0,001 \%$ to $2 \%^{2}$. The association between these anomalies has not previously been described. This case involved the management of a LGMD patient with an unexpected $\mathrm{Tb}$, which demanded an unexpected change in the lung insolation technique needed for the thoracoscopic sympathectomy.

\section{Learning points:}

Anesthetic management of neuromuscular disorders has several critical points to consider and unexpected/undescribed associations should be expected.

\section{References:}

1. Anestezlojogia i Ratownictwo 2013;7:397400

2. Otolaryngol Head Neck Surg 2002;126:2043 